\title{
Coverage and Throughput Analysis for FRET-Based Mobile Molecular Sensor/Actor Nanonetworks
}

\author{
Murat Kuscu*, Ozgur B. Akan \\ Next-generation and Wireless Communications Laboratory (NWCL) \\ Department of Electrical and Electronics Engineering \\ Koc University, 34450, Istanbul, Turkey
}

\begin{abstract}
Nanonetworks are envisaged to expand the capabilities of single nanomachines by enabling collaboration through communication between them. Förster Resonance Energy Transfer (FRET) observed among fluorescent molecules is a promising means of high-rate and reliable information transfer between single fluorophore-based nanoscale molecular machines. Recent theoretical studies have underlined its practicality for mobile ad hoc nanonetworks consisting of functionalized fluorescent molecules. In this study, we focus on the spatial characteristics of FRET-Based Mobile Molecular Sensor/Actor Nanonetworks (FRET-MSAN) by investigating the network performance in terms of communication coverage, network throughput and information propagation rate through extensive Monte Carlo simulations. The effect of fundamental system parameters related to FRET and to the mobility of the network nodes on the network performance are revealed. The results of the simulations indicate that the throughput and propagation rate as a function of distance from the information source are well-fitted by exponential curves. We also observe that the impact of FRET mechanism suppresses the effect of Brownian motion of network nodes on the exciton mobility.
\end{abstract}

Keywords: FRET, nanocommunications, mobile molecular sensor/actor nanonetwork, coverage, throughput, exciton diffusion

\section{Introduction}

Nanomachines are envisioned as nano-sized functional units with basic operational capabilities such as computing, sensing, actuating. The limited capabilities of these tiny machines can be enhanced by enabling communication

\footnotetext{
* Corresponding author

Email addresses: mkuscu@ku.edu.tr (Murat Kuscu), akan@ku.edu.tr (Ozgur B. Akan)
} 
between them. Establishing collaborative nanonetworks among diversely specialized nanomachines is the key to realize advanced applications that nanotechnology promises such as continuous health monitoring, smart drug delivery and cancer therapy with molecular precision [1]. Several attempts have been devoted to design practical and reliable nanocommunication methods such as THz-band electromagnetic and bio-inspired molecular communications [2, 3]. Recently, we have proposed a radically different molecular communication mechanism exploiting the excited-state transfer phenomenon of fluorescent molecules, i.e., Förster Resonance Energy Transfer (FRET) [4].

FRET is the non-radiative resonant transfer of excitons from an excited donor fluorophore to a ground-state fluorophore based on the near-field Coulomb interactions between them [5. The phenomenon has been extensively used in biotechnological applications ranging from fluorescence microscopy to biosensing and nanomedicine [6, [7. FRET has been proposed as a communication mechanism for the first time in 4 based on the idea of encoding the information to the excited-states of fluorescent molecules, and its performance for several channel configurations such as point-to-point, broadcast and relay have been analyzed extensively in [8, [9].

Recently, we have studied FRET-based mobile molecular sensor/actor nanonetworks (FRET-MSAN) composed of bioluminescent fluorophores as the sensor nodes and photoactive florophores as the actor nodes which collaboratively perform a common task, and showed its potential for in-vivo automated biosensing/acting applications [10]. We have modeled the information transmission propagation in these mobile nanonetworks exploiting the SIR model of epidemic disease spreading, and derived analytical expressions for the successful transfer of sensing information to an actor node in the environment by assuming that the fluorophore nodes are rapidly diffusing and have a very long excited-state lifetimes. However, using the SIR model, we ignored the spatial characteristics of the information propagation which are crucial especially for in-vivo medical applications.

In this paper, we analyze the FRET-MSAN based on the fundamental performance metrics related to spatial characteristics of the network such as the communication coverage, the network throughput as a function of distance from the information source, and the propagation rate. The exciton mobility combined with the Brownian motion of network nodes are well-observed through realistic Monte Carlo simulations. The effect of the system parameters related both to the exciton transfer mechanism and to the diffusion of the nodes on the exciton mobility are clearly revealed from the simulation results. Through the discussions, we aim to pave the way for the practical design of applicationspecific sensor/actor nanonetworks by configuring the easily controllable system parameters.

The remainder of this paper is organized as follows. In Section II, we present the fundamentals of FRET theory. In Section III, we introduce the FRETMSAN and describe the analyzed network scenario. The performance analysis based on the simulation results is presented Section IV. Finally, the concluding remarks are given in Section V. 


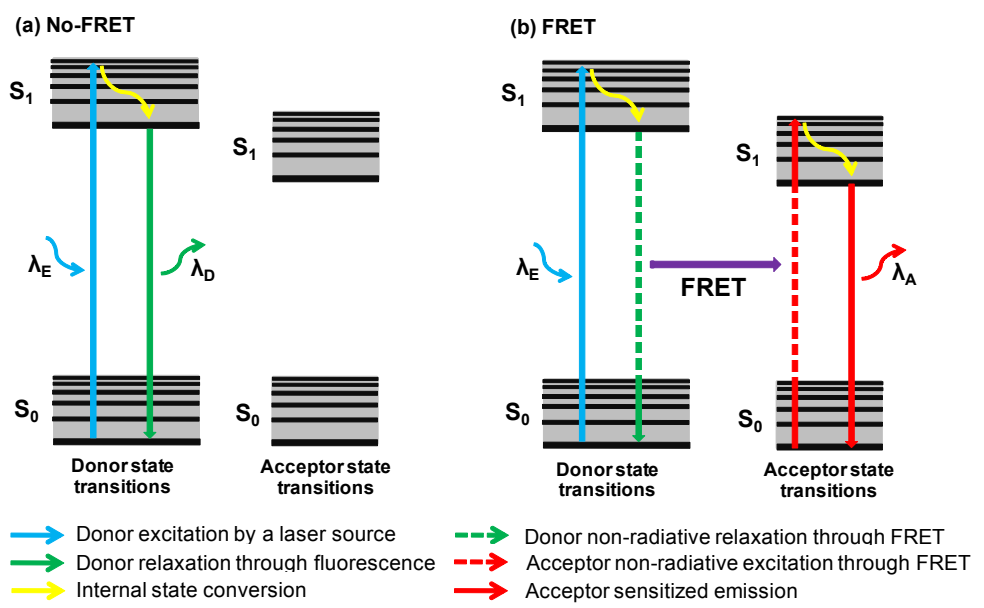

Figure 1: Jablonski diagram demonstrating the energy flow for the cases of (a) fluorescence and (b) FRET.

\section{Theory of FRET}

FRET phenomenon has a well-established theory which is fundamentally validated by an extensive number of experimental studies over the years [1]. In this section, we present the governing rate equations and briefly introduce the FRET mechanism.

When a fluorescent molecule is excited through an optical source, it relaxes after a random time either through fluorescing, i.e., releasing a photon, or through a non-radiative quenching mechanism. The natural fluorescing rate of a fluorophore $k_{0}$ is the reciprocal of its mean excited-state lifetime, i.e., $k_{0}=1 / \tau_{0}$. The lifetime denotes the average time that the molecule stays in the excitedstate before relaxing to the ground-state, and it is generally in the range of $2 \mathrm{~ns}-1 \mu \mathrm{s}$ [5]. There are several quenching mechanisms that can shorten the lifetime of an exciton on a fluorophore such as collisional quenching and transfer to a nearby ground-state fluorophore through FRET. Fig 1 demonstrates the Jablonski diagram for the case when FRET is the only possible quenching mechanism.

Mainly two conditions are required for FRET to occur. The spectral characteristics of donor, which is the excited fluorophore, and acceptor in the groundstate must have significant similarity, i.e., the emission spectrum of the donor and the absorption spectrum of the acceptor must overlap. The spectral similarity is proportional to the resonance probability of the molecules' transition dipoles, therefore, it increases the transfer rate. Moreover, the molecules must be in a close proximity such as $0-10 \mathrm{~nm}$. When these conditions are satisfied, FRET competes with fluorescence and other quenching processes. FRET rate between a pair of excited donor and a ground-state acceptor molecule with the 
intermolecular distance $R$ is given by

$$
k_{T}=k_{0}\left(\frac{R_{0}}{R}\right)^{6}
$$

where $R_{0}$ is the Förster radius denoting the distance between the molecules when $k_{T}=k_{0} . \quad R_{0}$ relates the FRET rate with environmental and intrinsic parameters and can be expressed by

$$
R_{0}=\left(8.8 \times 10^{22} \kappa^{2} n^{-4} Q_{D} J\right)^{-\frac{1}{6}}
$$

where $\kappa^{2}$ is the dipole orientation factor, $Q_{D}$ is the quantum yield of the donor and $n$ is the refractive index of the medium. $J$ denotes the degree of overlap between the donor's emission spectrum and the acceptor's absorption spectrum, and it is given by

$$
J=\int_{0}^{\infty} f_{D}(\lambda) \epsilon_{A}(\lambda) \lambda^{4} \mathrm{~d} \lambda
$$

where $f_{D}$ is the normalized emission spectral function of the donor, $\epsilon_{A}$ is the acceptor's molar absorptivity, and $\lambda$ is the wavelength. The dipole orientation factor $\kappa^{2}$ is expressed by

$$
\kappa^{2}=\left(\cos \theta_{T}-3 \cos \theta_{D} \cos \theta_{A}\right)^{2}
$$

where $\theta_{T}$ is the angle between the donor's emission dipole and the acceptor's absorption dipole moment, $\theta_{D}$ and $\theta_{A}$ are the angles between these dipole moments and the axis where donor and acceptor jointly located [5. It is commonly assumed that $\kappa^{2}=2 / 3$ which is the mean value when the molecules have isotropic and unrestricted distributions.

\section{FRET-Based Mobile Molecular Sensor/Actor Network (FRET- MSAN)}

The sensor/actor nanonetwork considered in this paper consists of single fluorophore-based sensor and actor nodes which realize three dimensional random movements based on Brownian motion. The aim of this nanonetwork is to execute a proper action with the actor nodes upon the detection of a target molecule or an event by the fluorescent sensor nodes. This advanced task which can be realizable by the collaboration of differently specialized fluorophore nodes exceeds the limits of a single fluorophore's capabilities, and constitutes an excellent example for the future applications of nanocommunications.

The fluorescent sensor nodes employed in this scenario are assumed to change their spectral characteristics when they bind to a specific target molecule. We also assume that the whole system is illuminated by an optical source which continuously releases photons with a certain wavelength. The signaling mechanism of a fluorescent sensor node can be described as follows: (i) The absorption spectrum of the nanosensor does not overlap with the wavelength of the optical 


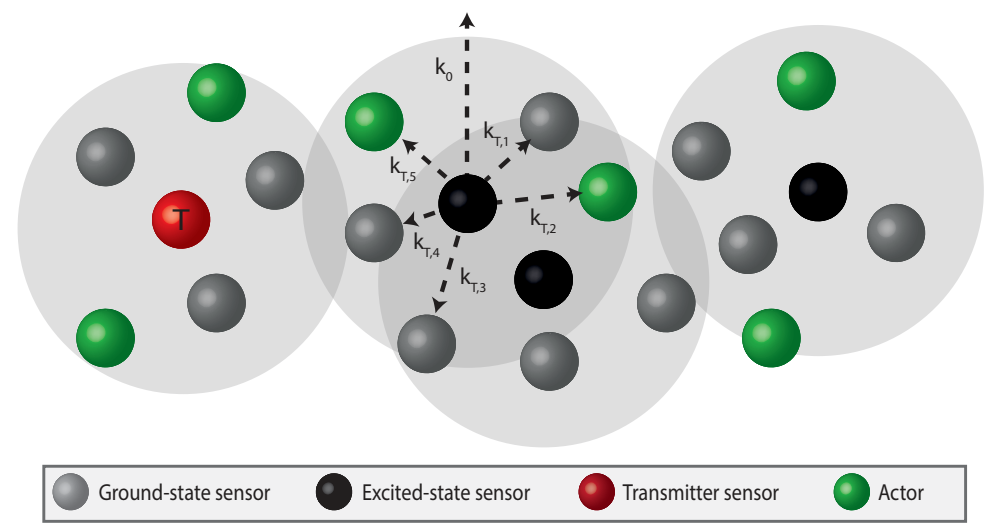

Figure 2: Two dimensional demonstration of an example FRET-MSAN. The arrows indicate the rates for exciton activity, and the circles denote the transfer range for an excited node.

source when it is not bound to any target molecule, therefore, it is not excited and stays in the ground-state. (ii) When the sensor node is bound to a target molecule, the resultant spectral shift causes an overlap of its absorption spectrum with the source wavelength, therefore, it becomes excited by absorbing a photon. After an exponentially random lifetime, it fluoresces indicating the existence of the target, if there is not any non-radiative quencher in its close vicinity. Through this mechanism, a sensor node acts as fluorescent switch which is turned on and off depending on the existence of a target molecule [12.

The actor nodes are assumed to consist of photosensitive molecules which are specialized to realize an action upon receiving an exciton. The photosynthesizing agents, e.g., porphyrin, used in the photodynamic therapy (PDT) of cancer are appropriate examples of single molecular exciton-activated actors [13. Under an illumination with a proper wavelength, these agents synthesize singlet oxygen which is extremely harmful for the living organisms, and therefore, they are used to initiate the apoptosis of the targeted cancer cells with the control of a remote optical excitation source. Recently, it has been experimentally proved that photosynthesizing agents can also be activated by FRET through a conjugation with a Quantum Dot (QD) [7].

In this study, we consider a scenario, demonstrated in Fig. 2, in which a single nanosensor, which we call as the transmitter sensor node, is bound to a target molecule. The transmitter is always in the excited-state during the application of the optical pulse, and the remaining sensor nodes stay in the ground-state since they are not bound to any target. The transmitter sensor node is assumed to be immobilized through the bound to the target molecule, while all the remaining nodes in the nanonetwork are mobile with Brownian motion. During the application of the excitation pulse with a length of $T_{p}>\tau_{0}$, many ground-state sensor and actor nodes can enter into proximity of the excited transmitter, therefore, they frequently quench it through FRET or collisions. 
As long as the optical pulse is applied, the transmitter is immediately excited after it is quenched to ground-state or relaxed through fluorescence, therefore, we expect that a high number of excitons are generated on the transmitter. If a generated exciton is transmitted to an actor node through FRET, it is assumed that the actor node immediately consumes the exciton to realize its action. When the generated excitons are received by the ground-state sensor nodes through homo-FRET, i.e., FRET between identical fluorophores [5]], they may remove the exciton from the system by relaxing through fluorescence or collisional quenching, or they may transfer it to another ground-state sensor node or to an actor node in their close vicinity. In a properly configured system, by the consecutive exciton transfers through the sensor nodes acting like relays, the detection information can spread to a considerable region, and many actor nodes can achieve to receive the information and collaboratively increase the impact of their action.

Since the nanonetwork nodes except the transmitter are mobile, the region covered by the excitons are expected to be larger compared to the network composed of immobile nodes. However, the Brownian motion of the network nodes can have an adverse effect on the mobility of excitons, since it also increases the rate of collisions between nodes and succeeding collisional quenchings, especially when the molecular density of the environment is high. At this point, to determine the effect of main system parameters related to FRET mechanism and Brownian process on the communication coverage and diffusion rate of excitons is very crucial to design a powerful application.

In our previous work [10, we have modeled the FRET-MSAN exploiting the Suspected-Infected-Recovered (SIR) model of epidemic disease spreading assuming that the network nodes are highly mobilized and have very long excited-state lifetimes which are greater than $1 \mu s$. We have achieved to derive closed-form expressions for the probability of a transmitter sensor node to successfully transmit its information to an actor node. However, using the SIR model, we have ignored the spatial characteristics of the information spreading which are very valuable for the applications like PDT. Since the main purposes of the cancer therapies including PDT are both to be effective on a focused region where the target is located, and not to harm the cells which function properly, the throughput of the designed system as a function of distance from the target is also important. However, to derive closed-form expressions for these spatial properties of the system is very difficult considering that the system has a very high degree of randomness which is resultant from the following characteristics:

- The sojourn times of excitons on the network nodes including the transmitter are exponentially random variables with mean values depending on the distance and the number of ground-state network nodes in the close vicinity.

- The excitons have random lifetimes on the system, and the probability for each exciton to be removed from the system by collisional quenching, fluorescing, or reception by an actor node at each time instant varies depending on the system state. 
- The network nodes, that constitute the lattice for the excitons' random jumps, randomly move based on Brownian dynamics, and the state of the nodes, e.g., excited or ground-state, frequently changes with the random jump of the excitons.

Due to the difficulty to analytically model the spatial properties of the system, in the next section, we design a Monte Carlo algorithm to realistically simulate the information propagation on the system, and reveal the main characteristics like communication coverage, throughput, and propagation rate for varying system parameters.

\section{Performance Analysis}

We conduct extensive Monte Carlo simulations to determine the effect of fundamental system parameters on the characteristics of exciton mobility in FRET-MSAN. In this section, we describe the simulation setup and present the results with discussions.

\subsection{Simulation Setup}

The simulations are performed in MATLAB by dividing the whole process into small time intervals and assuming that an exciton realizes only one activity, e.g., FRET, fluorescence, during a simulation timestep. The simulation algorithm is demonstrated in Fig. 3 . The following list summarizes the assumptions made and governing equations for the simulations:

1. The excited-state lifetimes $\tau_{0}$ and the diffusion constant $D$ of all the nodes are assumed to be identical. The Förster radii between each network node $R_{0}$ are also taken as the same.

2. The simulation timestep is determined in proportion to the excited-state lifetime of the network nodes, $\Delta t=0.001 \tau_{0}$, to almost guarantee no more than single exciton activity in a timestep.

3. The initial positions of the network nodes are randomly determined for each run of simulation following a uniform probability distribution on a three dimensional cubic lattice with edge length of $400 \mathrm{~nm}$. Periodic boundary conditions are applied for the motion of network nodes.

4. The transmitter sensor node which is bound to a point target, is always in the excited-state during the application of optical pulse.

5. The length of the optical pulse $T_{p}$ is assumed to be 200ns, and the simulations are run for a duration of 250ns which almost ensures that all of the generated excitons are removed from the system at the end of the simulation.

6. The dipole orientation factor $\kappa^{2}$ is taken as $2 / 3$ which is the average value assuming free rotation of network nodes. 


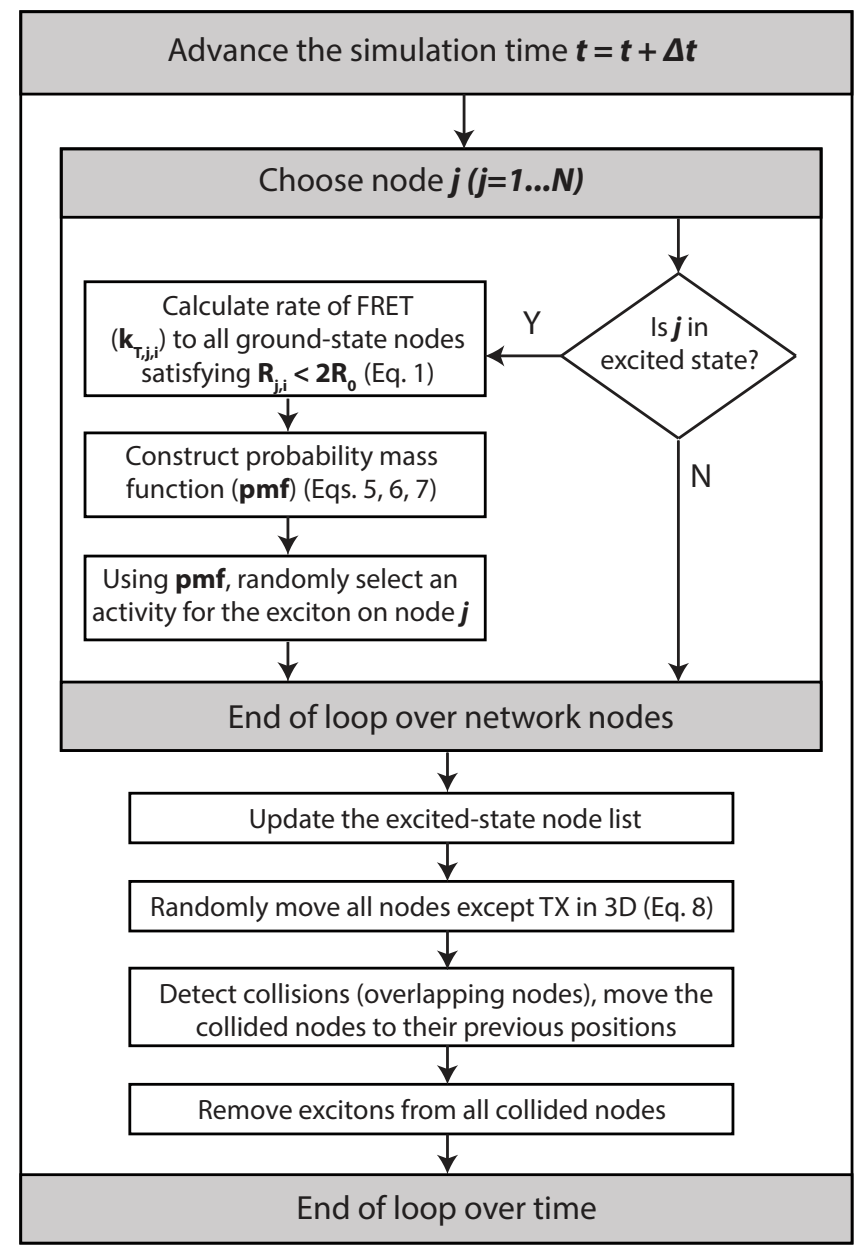

Figure 3: Simulation algorithm for the exciton jump process on the Brownian mobile nodes. Simulation ends when $t=250 \mathrm{~ns}$.

7. The network nodes are modeled as non-interacting hard spheres with diameters $d=1.5 \mathrm{~nm}$.

8. FRET rate between two node becomes very low when the internodal distance exceeds $2 R_{0}$. Therefore, in the simulations, the transfer range for an excited node is assumed to be $2 R_{0}$; hence, the transfer probabilities to the nodes which are located at distances greater than $2 R_{0}$ from the excited node are not calculated.

9. The transition probabilities for an exciton occupied on a sensor node given that the node does not collide with another network node during 
a timestep are as follows:

(i) The transfer probability to a ground-state sensor node or to an actor node:

$$
P_{j, i}=k_{T, j, i} \Delta t \quad i=1, \ldots, k
$$

where $j$ is the index of the occupied node, $i$ is the index of the node to be transferred, and $1, \ldots, k$ are the indices of ground-state, i.e., available, sensor and actor nodes in the transfer range of occupied node. $k_{T, j, i}$ is the transfer rate from node $j$ to node $i$, and calculated using (1).

(ii) The probability of removal through fluorescence:

$$
P_{j, 0}=k_{0} \Delta t
$$

(iii) The probability of remaining at the same state, i.e., on the same node:

$$
P_{j, j}=1-P_{j, 0}-\sum_{i=1}^{k} P_{j, i}
$$

10. All of the network nodes except the transmitter are assumed to realize random motion. We use Brownian dynamics which is computationally inexpensive to describe their movement and neglect the acceleration of the nodes. Their positional increments at each timestep are randomly assigned according to the following normal distribution [14]:

$$
d x_{\Delta t}=d y_{\Delta t}=d z_{\Delta t}=\mathcal{N}(0, \sqrt{2 D \Delta t})
$$

where $D$ is the diffusion coefficient of the network nodes, and $d x, d y, d z$ are the positional increments through $x, y, z$ directions, respectively.

11. Since we neglect the acceleration of the nodes, the collision has to be described in a simplified manner. If an overlap between network nodes is detected as a result of their motion, the nodes are assumed to collide and move back to their position at the previous timestep. If any of the collided nodes are in the excited-state, the excitons are removed from the system as a consequence of collisional quenching. At each timestep, this is repeated until there is no overlapping node in the system.

The simulations are conducted for varying system parameters such as the excited-state lifetime, the diffusion coefficient, the Förster radii, and the concentration of molecules, and repeated for each configuration until the observed parameters converge to finite values $(\sim 10000$ repetitions for each parameter change). The default values for the main system parameters are listed in Table 1. 
Table 1: Simulation Parameters for FRET-MSAN

\begin{tabular}{l|l}
\hline \hline Excited state lifetime $\left(\tau_{0}\right)$ & $10 \mathrm{~ns}$ \\
\hline Förster radius $\left(R_{0}\right)$ & $6 \mathrm{~nm}$ \\
\hline Total molecular concentration $\left(C_{T}\right)$ & $1.44 \mathrm{mM}$ \\
\hline Concentration ratio $\left(C_{A} / C_{S}\right)$ & 1 \\
\hline Diffusion constant $(D)$ & $0.4 \mathrm{~nm}^{2} / \mathrm{ns}$ \\
\hline
\end{tabular}

\subsection{Results}

The throughput of the FRET-MSAN is the overall transfer rate of information to the actor nodes, and it is equal to the rate at which the actor nodes perform their task, e.g., production of singlet oxygen in a PDT application. Therefore, throughput is one of the fundamental performance metrics. In the Monte Carlo simulations, we observe the number of actor node excitations with the spatial information at the resolution of $4 \mathrm{~nm}$ for different diffusion coefficients, Förster radii, excited-state lifetimes and molecular concentrations. The throughput $T$ as a function of distance from the transmitter is calculated as the ratio of the excitations of the actor nodes located outside the sphere with radius $R$ and centered at the transmitter's location to the excitation pulse length $T_{p}=200 \mathrm{~ns}$. The results also reveal the network coverage, i.e., the region where the throughput is focused on.

We also observe the mean first passage times of excitons $T_{f}$ through the spheres with radius $R$ and centered at the transmitter's location to reveal the propagation rate of the detection information. All of the simulated parameters significantly affect the performance, and in the following sections, we discuss the reasons behind each outcome. It is to be noted that, the obtained throughput and first passage time data as a result of extensive number of simulations are well-expressed with decreasing and increasing exponential functions, respectively. In each plot demonstrating the outcomes for different parameters, we also give the well-fitted exponential curves.

\subsubsection{Effect of Diffusion Constant}

For the first analysis, we observe the effect of node mobility on the throughput, coverage, and propagation rate. The diffusion constant $D$ is varied in a range between $0.1-0.1 \mathrm{~nm}^{2} / \mathrm{ns}$, which is common for fluorescent molecules in aqueous environments [5]. The simulation results for throughput in Fig. 4(a)] are quite interesting, since the high mobility of network nodes, i.e., the increased diffusion constant, results in a decrease of the throughput and the effective range. Actually, the process of exciton transfer is very fast compared to the Brownian motion of the nodes, therefore, the slightly increased node mobility cannot effectively increase the exciton mobility. Moreover, the higher collision rate with the greater diffusion constant, results in higher rate of collisional quenching of excitons, therefore, it decreases the number of excitons on the system averaged over time, and results in narrower coverage. We reveal from the results that the 


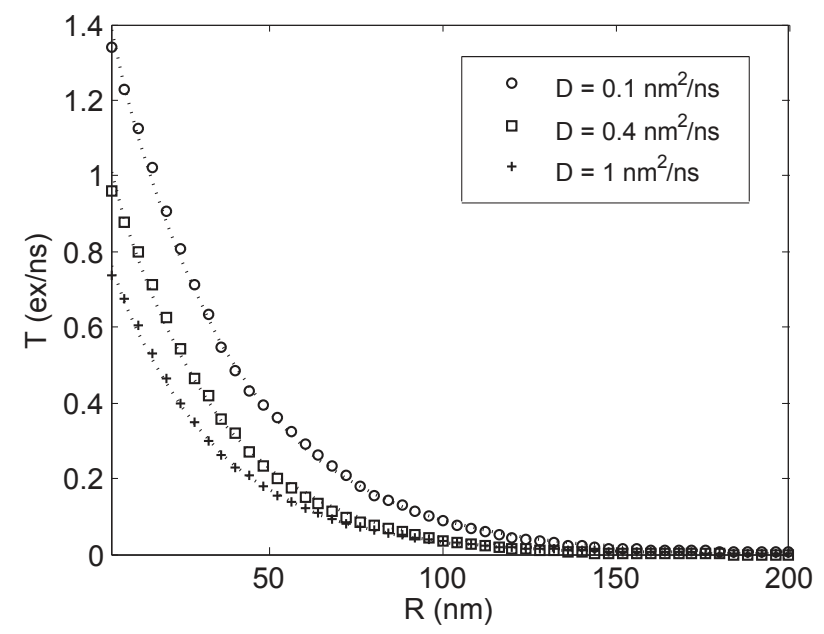

(a) $T$ with varying $R$ for different $D$.

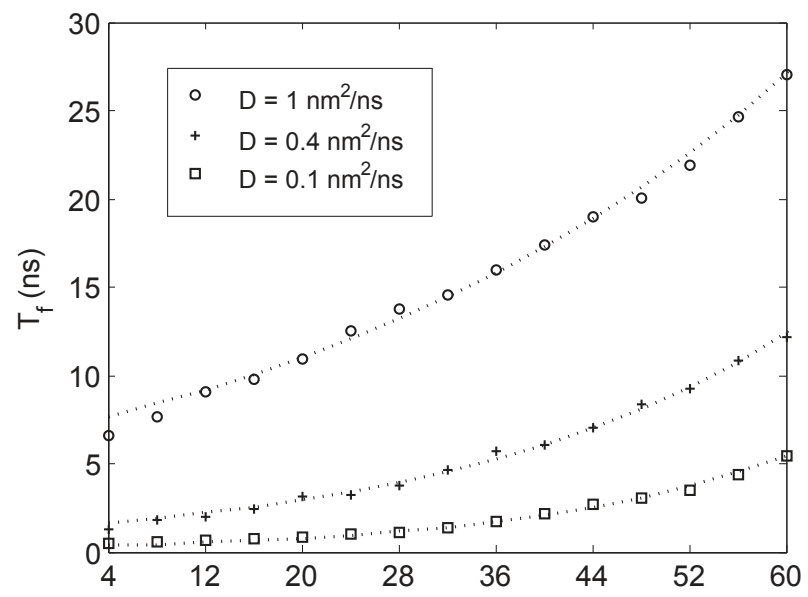

(b) $T_{f}$ with varying $R$ for different $D$.

Figure 4: The mean network throughput $T$ and mean first passage times of excitons $T_{f}$ as a function of $R$ for varying diffusion constant $D$ with fitted exponential curves.

exciton activity is effective up to $100 \mathrm{~nm}$-distance from the transmitter while $D=0.1 \mathrm{~nm}^{2} / \mathrm{ns}$.

The same reasons are valid for the increased mean first passage times when the diffusion constant gets greater, as shown in Fig 4(b). Since the average number of excitons on the system decreases with the increasing collisional quenching rate, the mean time for the smaller number of excitons that achieve surviving 


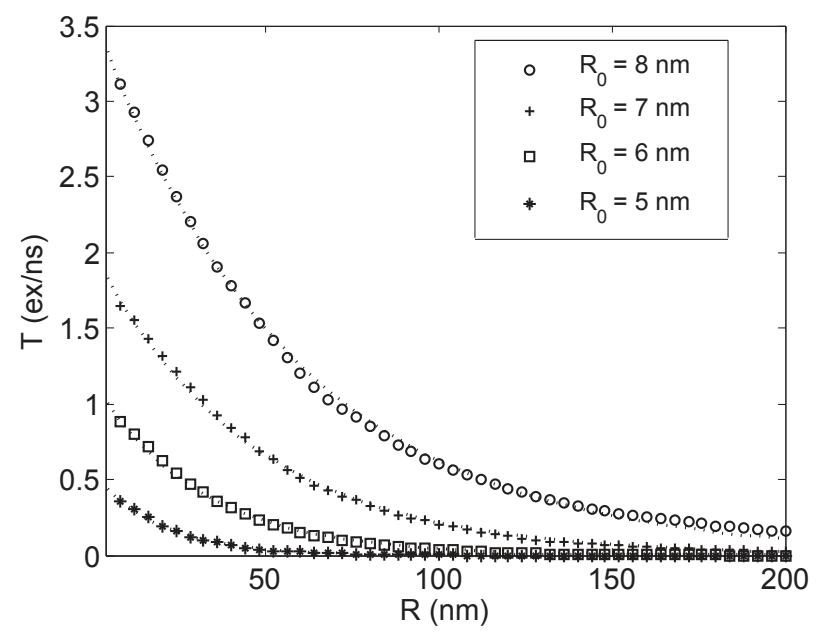

(a) $T$ with varying $R$ for different $R_{0}$.

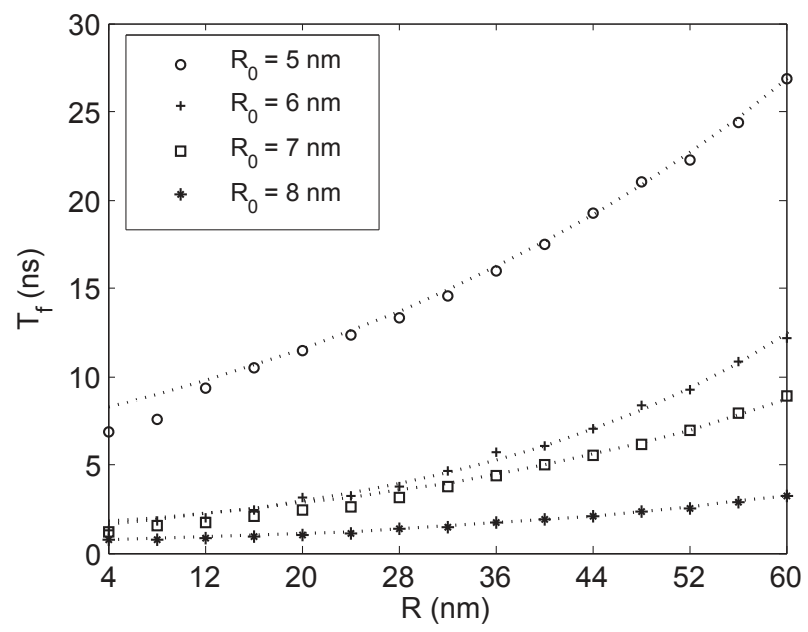

(b) $T_{f}$ with varying $R$ for different $R_{0}$.

Figure 5: The mean network throughput $T$ and mean first passage times of excitons $T_{f}$ as a function of $R$ for varying Förster radius $R_{0}$ with fitted exponential curves.

to reach a certain distance from the transmitter also increases.

\subsubsection{Effect of Förster Radius}

Fig. 5(a) demonstrates the effect of Förster radius $R_{0}$ on the throughput performance of the network. As is seen, the higher $R_{0}$ results in higher throughput and significantly larger coverage. The increased $R_{0}$ extends the transfer range 
for a single exciton. Moreover, the FRET rate is strongly enhanced with the increased $R_{0}$. As a consequence, excitons are more frequently generated on transmitter, and more frequently transferred between sensor nodes. Therefore, the chance for an exciton to survive at larger distances increases, and the chance for an actor node to receive an exciton through FRET from an excited sensor also increases which results in higher throughput for greater $R_{0}$.

The propagation rate is also strongly affected by the varying $R_{0}$ as is seen in Fig. 5(b) The high exciton mobility obtained by increasing $R_{0}$ significantly decreases the mean first passage time of excitons through certain distances. For $R_{0}=8 \mathrm{~nm}$, in a very short time less than $10 \mathrm{~ns}$, the volume of radius $60 \mathrm{~nm}$ is covered by the excitons signaling the existence of the target.

\subsubsection{Effect of Excited State Lifetime}

The excited-state lifetime $\tau_{0}$ strongly affects the throughput as seen in Fig. 6(a) As the lifetime increases, the network throughput significantly decreases. This is mainly resultant from the fact that when the lifetime increases, the exciton generation rate on the transmitter decreases, since each generated exciton stays longer time on transmitter. Moreover, the FRET rate decreases with greater sojourn times, and therefore, at each timestep, the probability for an exciton to be removed from the system through collisional quenching or fluorescence increases.

The increased lifetime also decreases the propagation rate of the excitons as demonstrated in Fig. 6(b) In fact, most of the simulations for $\tau_{0}=50$ ns resulted with the extinction of excitons before reaching to $60 \mathrm{~nm}$-barrier. Therefore, it is a wise strategy to employ short-lifetime molecules for applications requiring fast information dissemination over greater volumes.

\subsubsection{Effect of Molecular Concentration}

In order to investigate the impact of nodal density on the throughput and propagation rate, we simulate the system increasing the total molecular concentration and by keeping the individual concentrations of sensor and actor nodes as equal to each other. The results presented in Fig. 7(a) are not considerably different from the expected, such that, the throughput increases for the denser network. In fact, the higher concentration causes a decrease of the average intermolecular distances between network nodes, which results in the higher transfer rate, therefore, the exciton mobility increases. The exciton generation rate at the transmitter node is also increases, since the generated excitons are more frequently transmitted to the system through energy transfers. On the other hand, the increased nodal density causes higher collisional quenching rate. However, this time, the increased quenching rate cannot suppress the increase in the exciton mobility.

The result of the simulations for the propagation rate, given in Fig. 7(b) clearly demonstrates that when the concentration is lower, it takes longer time on average to reach a certain distance from the transmitter, underlining the reduced exciton mobility resultant from the decreased density. 


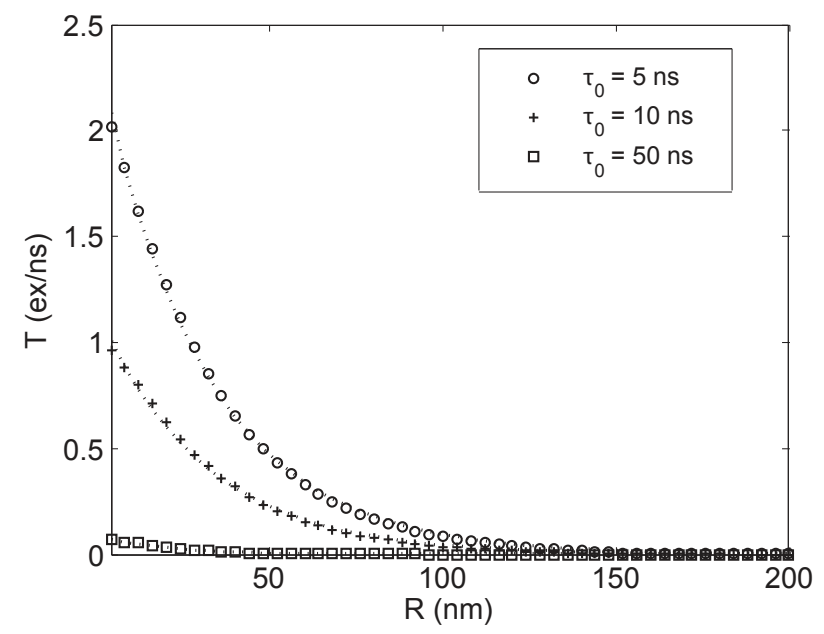

(a) $T$ with varying $R$ for different $\tau_{0}$.

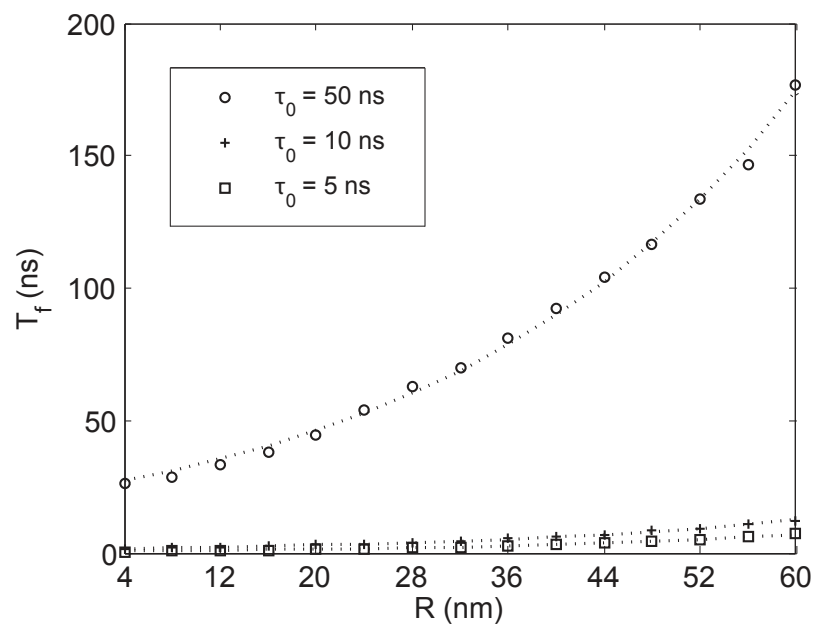

(b) $T_{f}$ with varying $R$ for different $\tau_{0}$.

Figure 6: The mean network throughput $T$ and mean first passage times of excitons $T_{f}$ as a function of $R$ for varying mean excited state lifetime $\tau_{0}$ with fitted exponential curves.

\section{Conclusion}

In this paper, we analyze the fundamental spatial properties of information propagation in FRET-MSAN performing extensive Monte Carlo simulations. We investigate the effect of main system parameters on the coverage, network throughput and propagation rate, and conclude that the exciton mobility can be described by the FRET mechanism rather than the diffusion of network nodes. 


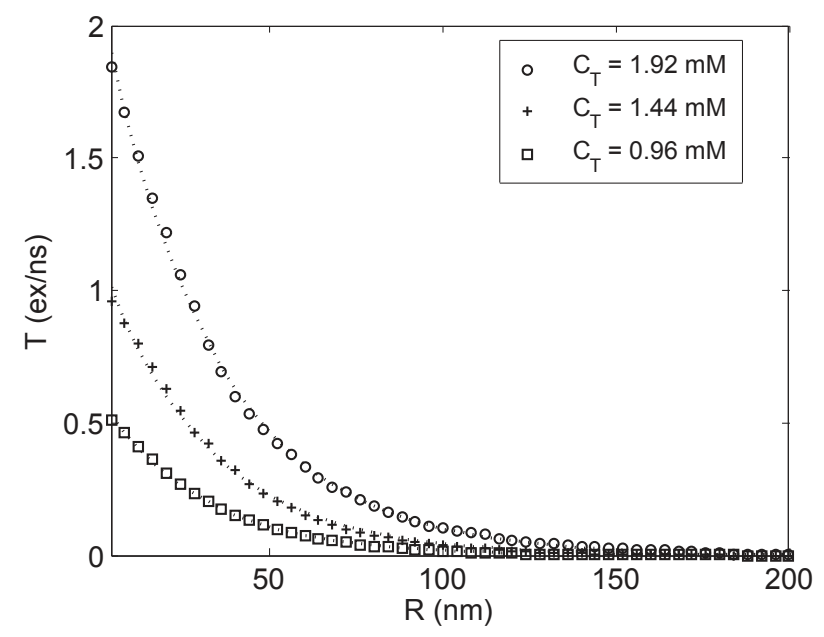

(a) $T$ with varying $R$ for different $C_{T}$.

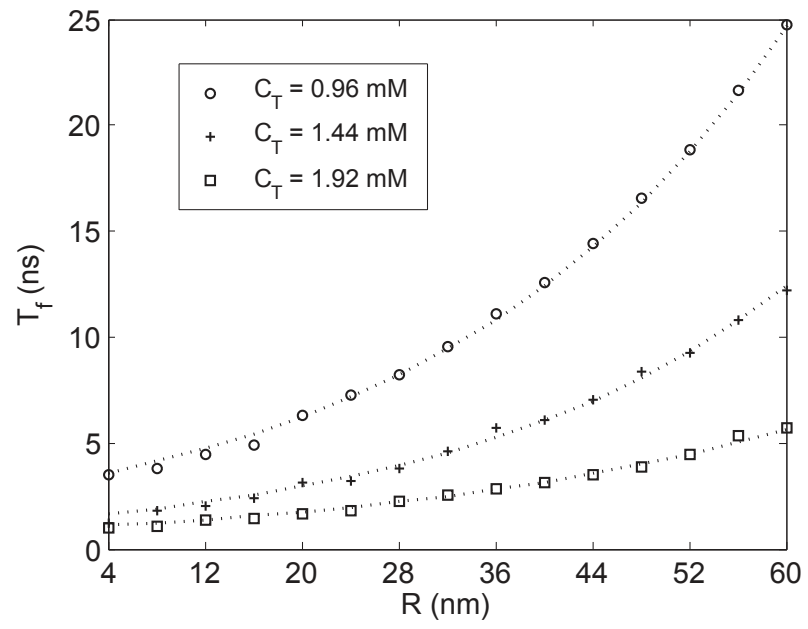

(b) $T_{f}$ with varying $R$ for different $C_{T}$.

Figure 7: The mean network throughput $T$ and mean first passage times of excitons $T_{f}$ as a function of $R$ for varying total concentration $C_{T}$ with fitted exponential curves.

We show that excitons signaling the existence of a single target molecule can rapidly propagate and activate actor nodes over significant distances compared to their sizes when the system parameters are properly set. 


\section{Acknowledgment}

This work was supported in part by the Turkish Scientific and Technical Research Council Career Award under grant \#109E257 and by the Turkish National Academy of Sciences Distinguished Young Scientist Award Program (TUBA-GEBIP) and by IBM through IBM Faculty Award.

\section{References}

[1] I. Akyildiz, F. Brunetti, C. Blazquez, Nanonetworks: A new communication paradigm, Computer Networks 52 (12) (2008) 2260-2279. doi: $10.1016 / \mathrm{j}$.comnet.2008.04.001.

[2] I. F. Akyildiz, M. J. Jornet, Electromagnetic wireless nanosensor networks, Nano Communication Networks 1 (1) (2010) 3-19. doi:10.1016/ j.nancom.2010.04.001.

[3] T. Nakano, T. Suda, M. Moore, R. Egashira, A. Enomoto, K. Arima, Molecular communication for nanomachines using intercellular calcium signaling, in: Proceedings of IEEE NANO 2005, Nagoya, Japan, 2005.

[4] M. Kuscu, O. B. Akan, A physical channel model and analysis for nanoscale molecular communications with forster resonance energy transfer (fret), IEEE Transactions on Nanotechnology 11 (1) (2012) 200-207. doi:10. 1109/TNANO.2011.2170705.

[5] J. R. Lakowicz, Principles of fluorescence spectroscopy, 3rd Edition, Springer-Verlag, Baltimore, MD, 2006.

[6] E. A. Jares-Erijman, T. M. Jovin, Fret imaging, Nature Biotechnology 21 (2003) 1387-1395. doi:10.1038/nbt896

[7] A. Samia, X. Chen, C. Burda, Semiconductor quantum dots for photodynamic therapy, Journal of the American Chemical Society 125 (51) (2003) 15736-15737. doi:10.1021/ja0386905.

[8] M. Kuscu, D. Malak, O. Akan, An information theoretical analysis of broadcast networks and channel routing for fret-based nanoscale communications, in: Proceedings of IEEE MoNaCom 2012, Ottawa, Canada, 2012.

[9] M. Kuscu, O. Akan, Multi-step fret-based long range nanoscale communication channel, IEEE Journal on Selected Areas in Communications (JSAC) 31 (12) (2013) 715-725. doi:10.1109/JSAC. 2013.SUP2.1213004.

[10] M. Kuscu, O. B. Akan, Fret-based mobile molecular nanonetworks, in: Proceedings of IEEE Med-Hoc-Net 2013, Ajaccio, Corsica, France, 2013.

[11] T. Forster, Zwischenmolekulare energiewanderung und fluoreszenz, Annalen der Physik 437 (1948) 55-75. doi:10.1002/andp.19484370105. 
[12] A. Silva, D. Fox, T. Moody, S. Weir, The development of molecular fluorescent switches, Trends in Biotechnology 19 (1) (2001) 29-34. doi: 10.1016/S0167-7799(00)01513-4.

[13] R. Bonnett, Photosensitizers of the porphyrin and phthalocyanine series for photodynamic therapy, Chemical Society Reviews 24 (1995) 19-33. doi: 10.1039/CS9952400019.

[14] E. Cinlar, Introduction to Stochastic Processes, Dover, Mineola, NY, 2013. 